\title{
Survey on different methods in image compression of Brain Images
}

\author{
Sandip Rajendra Udawant ",", Satyawati Magar ${ }^{\text {a }}$ \\ " Department of E \& TC, D.V.V.P College of Engineering, Ahmednagar, Maharashtra, India. \\ *Corresponding Author: udawant_etc@enggnagar.com
}

Received : $12^{\text {th }}$ June 2020, Accepted : $26^{\text {th }}$ August 2020

\begin{abstract}
The survey of brain and medical image compression methods. Reduce the size of image as image compression. Necessity and importance of compression of an image has been discussed. Application of the lossy compression technique is multimedia data. Various compression approaches are discussed for two categories. Also brain image compression techniques are highlighted, in addition with, quantitative comparisons between different compression methods. Also advantages and disadvantages of each method are discussed.
\end{abstract}

Keywords: Medical Image Compression, Brain Image, Medical Images, CR, PSNR, DWT, DCT \& DFT

\section{Introduction}

This paper describes that for compression of an image lossy compression method is not that much useful. Due to compression method there is possibility of loss in useful information, which has been used for researchers and practitioners. Some operations like enhancement may steer to further deterioration hence there is need of efficient method of image compression. Lossless compression as a better option as a remedy. Many lossless schemes are based on linear prediction and interpolation. There are exploitations of correlation within the image frame, hence context based approach is used popularly. This paper brief about the various coding algorithms \& betterment as well as advancement in this field.

Now a days various image compression techniques are used by researchers. For achieving better results than existing techniques there is need to develop new Hybrid technique and algorithms. 
Vol. 2 Iss. 2 Year 2020 Sandip Rajendra Udawant \& Satyawati Magar /2020

\section{Survey \& finding}

A) A Survey On Coding Algorithms In Medical Image Compression by S. Bhavani \& Dr. K. Thanushkodi, International Journal of Computer Science \& Engineering.

\section{Findings:}

1) Lossy compression methods is not that much useful.

2) Due to lossy compression method there is possibility of loss in useful information.

3) Some operations like enhancement may steer to further deterioration hence there is need of efficient method of image compression.

4) Lossless compression used as a remedy.

5) Various coding algorithms and betterment as well as advancement in this field are discussed.

B) An Overview of Image Compression Approaches by Cebrail Taskin, Serdar Kursat Sarikoz, The Third International Conference on Digital Telecommunications.

\section{Findings:}

1) The purpose of both compression techniques i.e. lossy and lossless.

2) These techniques are used to reduce the size of the Compressed image size is affected by the ratio of the compression.

3) The ratio of lossless image compression is $2: 1$ or 3:1. and the ratio varies from 10:1 to 50:1 for the lossy compression.

C) Brain Image Compression Techniques by M.Abo-Zahhad, R.R.Gharieb, Sabah M.Ahmed, Mahmoud Khaled, International Journal of Engineering Trends and Technology (IJETT).

\section{Findings:}

1) Brain and medical image compression has discussed.

2) Necessity and importance of compression of an image has been discussed.

Application of the lossy compression technique is multimedia data such as audio, video, and still images. 
Vol. 2 Iss. 2 Year 2020 Sandip Rajendra Udawant \& Satyawati Magar /2020

D) An Overview of Principal Component Analysis by Sasan Karamizadeh, Information Processing

\section{Findings:}

1) Importance of PCA in image processing has been discussed.

2) Mainly the basic steps of PCA algorithm are elaborated, which are useful for implementation in the image processing.

E) A New Method of Image Quality Assessment by Shuang Liang, Guanxiang Wang, Shuli Wang, Yu Wang

WSEAS TRANSACTIONS on Signal Processing.

\section{Findings:}

1) The quality measurement and assessment i.e. MSE, PSNR Structure-WSNR, NQM, UQI, VSNR, SSIM.

2) Natural scene images-VIF, IFC are elaborated.

3) Also brief discussion has been carried out on image texture.

4) Image quality assessment based on brightness discrimination, error density and gradient (BDEDG).

F) Assessment The Quality of Medical Images (CT and MRI) by Using Wavelet Transformation (WT) by Ziad M. Abood

International Journal of Emerging Research in Management and Technology.ISSN:

\section{Findings:}

1) The parameters are MSE, MAE, PSNR., SC, MD, AD, SC, LMSE, NAE, RMSE, PSME, IF, NK, CQ, UQI, SSIM, FSIM, SR-IM, RFSIM,VIF, SNR, VSNR and PCC has been discussed in detail.

2) Importance of quality measurement has been discussed.

G) Image Compression Using Discrete Wavelet Transform and Discrete Cosine Transform by Dr. Taif Sami Hasan,

Journal of Applied Science and Research 
Vol. 2 Iss. 2 Year 2020 Sandip Rajendra Udawant \& Satyawati Magar /2020

\section{Findings:}

1) Simple and efficient algorithm were discussed by using DCT \& DWT.

2) DCT gives excellent $\mathrm{CR}$ as well as image quality.

3) Wavelet transform is very superior method, for both transform JPEG image is considered.

4) Four section of image are done in wavelet transform which are $\mathrm{CA}, \mathrm{CH}, \mathrm{CV}$, and $\mathrm{CD}$.

5) Image is divided into blocks each $8 \times 8$ in size and then applied discrete cosine transform (DCT), quantization process, zigzag and run length encoding (RLE).

H) Fractal Based Image Compression Techniques by Sandhya Kadam, Vijay Rathod by International Journal of Computer Applications.

\section{Findings:}

1) Fractal image compression, which offers high compression ratios and quality image reconstruction.

2) Main highlights of this paper are the different issues in fractal image compression as partitioning, larger encoding time, compression ratio, quality of the reconstructed image, decoding time, SSIM (Structured Similarity Index).

3) Areas for improvement are suggested in this article are larger encoding time and PSNR.

4) Evaluating different parameters viz. PSNR, compression ratio, encoding time, and decoding time are also suggested.

I) Neural Based Domain And Range Pool Partitioning Using Fractal Coding For Nearly Lossless medical Image Compress by S.Bhavani \& Dr.K.Thanushkodi, WSEAS Transactions on signal Processing, Issue

\section{Findings:}

1) In this work improved quasi-lossless fractal coding scheme is described which preserve the rich features of the medical images.

2) To improve the performance of the fractal coding and to reduce encoding computational complexity machine learning model is used.

3) To evaluate the performance Compression Ratio (CR), PSNR and encoding computational time are considered for MRI image. 
J) A Review of Image Enhancement Techniques in Image Processing by Gaurav Mohan Singh, Mahipal Singh Kohliyand Manoj Diwakar,

\section{Findings:}

1) Study of Histogram equalization (HE), adaptive histogram equalization (AHE), and Decorrelation stretch (DCS).

2) Quality and contract of entire image is done by multichannel image enhancement technique.

3) Image contrast can be improved by image enhancement technique. This paper concluded that better results of image contrasts are obtained by $\mathrm{HE}$.

K) Contrast Limited Adaptive Histogram Equalization Image Processing to Improve the Detection of Simulated Spiculations in Dense Mammograms by Etta D. Pisano Journal of Digital Imaging.

\section{Findings:}

1) Study and use of CLAHE is discussed.

2) By using "Contrast Limited Adaptive Histogram Equalization (CAHE)" results are improved.

3) CLAHE enhances the visibility of structures that extend across pixel boundaries.

L) A Survey of Medical Image Compression Using Hybrid Techniques by Mr.Amit S.Tajne, International Journal of Computer Science and mobile Computing

\section{Findings:}

1) Some unique characteristics are used.

2) All compression techniques are useful for real time medical image transmission and storage. Selection of high PSNR value will maintain the quality of the image and achieve successful results of compression process.

M) A Hybrid Scheme Coding Using SPIHT and Fractal for Mammography Image Compression by Benamrane Nacera Bentorki Soumia, 15th International Conference on Information Visualization. 
Vol. 2 Iss. 2 Year 2020 Sandip Rajendra Udawant \& Satyawati Magar /2020

\section{Findings:}

1) Study of medical imaging techniques are carried out. Due to advancement in the medical field, large amounts of data are produced at the time of diagnosis.

2) Fractal image compression having lacuna that it is having high encoding time.

3) New optimization approach to reduce the time of fractal image compression. The approach is a hybridization and used the SPIHT algorithm and Jacquin-style coding scheme for the same.

N) Digital Image Compression Hybrid Technique Based on Block Truncation Coding and Discrete Cosine Transform by Nehal Markandeya, Sonali Patil

\section{Findings:}

1) ICTEI, Proposed Hybrid image compression method is developed based on BTC and DCT.

2) Basic approach is to achieve good quality of reconstructed image hence PSNR \& MSE are measured.

3) Magnification after comparison between the proposed hybrid method and the BTC \& DCT.

4) Hence authors are concluded that, the proposed hybrid method performs better than the BTC and DCT.

P) Detecting (ROI) in Digital Mammogram by using Morphological BPF by Ju Cheng Yang, Dong Sun Park IEEE 2004.

\section{Findings:}

1) Micro calcifications are early sign of breast cancer appear, which are difficult to detect due to their small size.

2) $\mathrm{MBF}$ is introduced to detect micro calcifications.

It describes a new method for the detection of microcalcifications by using morphological bandpass filter. Comparing to DWT method, this method is more accurate in positions and sizes of microcalcifications. 
Vol. 2 Iss. 2 Year 2020 Sandip Rajendra Udawant \& Satyawati Magar /2020

\section{Conclusion}

In the entire survey of 15 papers it's found that for betterment and to achieve efficient and accurate coding of the medical images hybrid technique is used. Available compression scheme gives a very high compression rate with some loss of quality which is considerable. For doctors, it is necessary to have high image quality in ROI. This study discusses a hybrid model of lossless compression in ROI \& the lossy compression has been used in remaining region. Computers are preferably used for keeping images. To store amount of data, space is issue. Sufficient space is required to send the data or data should be compressed. To dominate these disadvantages image compression techniques are used.

\section{References}

[1] S. Bhavani, K. Thanushkodi, A survey on coding algorithms in medical image compression, International Journal on Computer Science and Engineering, 2 (2010) 14291434 .

[2] C. Taskin, S.K. Sarikoz, (2008) An overview of image compression approaches, In 2008 The Third International Conference on Digital Telecommunications, 174-179.

[3] M. Abo-Zahhad, R.R. Gharieb, Sabah M. Ahmed, Mahmoud Khale, Brain Image Compression Techniques, International Journal of Engineering Trends and Technology, 19 (2015) 93-105.

[4] Sasan Karamizadeh, Shahidan M, Abdullah, Azizah A. Manaf, Mazdak Zamani, An Overview of Principal Component Analysis, Journal of signal and Information Processing, (2013) 173-175.

[5] Shuang Liang, Guanxiang Wang, Shuli Wang, Yu Wang, A New Method of Image Quality Assessment, WSEAS TRANSACTIONS on Signal Processing, 12 (2016) 94-101.

[6] Ziad M. Abood, Kadhim K. Kadhim. Assessment the Quality of Medical Images (CT and MRI) by Using Wavelet Transformation (WT), International Journal of Emerging Research in Management and Technology, 4 (2015) 98-108.

[7] T. S. Hasan, Image Compression Using Discrete Wavelet Transform and Discrete Cosine Transform, Journal of Applied Science and Research, 13 (2017) 1-8.

[8] Sandhya Kadam, Vijay Rathod, Fractal Based Image Compression Techniques, International Journal of Computer Applications, 178(2017) 11-18.

[9] S. Bhavani, K. Thanushkodi, Neural Based Domain and Range Pool Partitioning Using Fractal Coding for Nearly Lossless medical Image Compress, WSEAS Transactions on signal Processing, 9 (2013) 11-20.

[10] G.M. Singh, M.S. Kohli, M. Diwakar, A review of image enhancement techniques in image processing, Technology Innovations and Research, 5 (2013) 2321-4135. 
[11]Etta D. Pisano. Shuquan Zong, Bradley M. Hemminger, Marla DeLuca, R. Eugene Johnston, Keith Muller, M. Patricia Braeuning, Stephen M. Pizer, Contrast Limited Adaptive Histogram Equalization Image Processing to Improve the Detection of Simulated Spiculations in Dense Mammograms, Journal of Digital Imaging, 11 (1998) 193-200.

[12] Amit S. Tajne. Pravin S. Kulkarni, A Survey of Medical Image Compression Using Hybrid Techniques, International Journal of Computer Science and mobile Computing, 4 (2015) $18-23$.

[13] Benamrane Nacera, Bentorki Soumia, A Hybrid Scheme Coding Using SPIHT and Fractal for Mammography Image Compression, 15th International Conference on Information Visualization, IEEE Xplore, 2011.

[14] Nehal Markandeya, Sonali Patil, (2017) Digital Image Compression Hybrid Technique Based on Block Truncation Coding and Discrete Cosine Transform, International Conference on Trends in Electronics and Informatics ICEI, IEEE, 1159-1162.

[15]J. Yang, D. Park, (2004) Detecting region-of-interest (RoI) in digital mammogram by using morphological bandpass filter, In 2004 IEEE International Conference on Multimedia and Expo (ICME) (IEEE Cat. No. 04TH8763), IEEE, 2 (2004) 1279-1282.

[16] R.N. Chaudhary, (2006) Waves and Oscillations, New Edge International Publishers, India.

[17] Stephan Chaphman, (2012) Matlab Programming for Engineers, Cengage Learning Publishers, USA.

\section{Acknowledgements: NIL}

\section{Conflict of interest: NIL}

About the License: (C) 2020 The Authors. This work is licensed under a Creative Commons Attribution 4.0 International License which permits unrestricted use, provided the original author and source are credited. 